\title{
ELECTROWEAK PENGUIN AND LEPTONIC DECAYS AT BABAR
}

\author{
F. BUCCI \\ Università di Pisa and INFN Sezione di Pisa, \\ Via Filippo Buonarroti, 2 - 56127 Pisa, Italia \\ E-mail: francesca.bucci@pi.infn.it
}

\begin{abstract}
Recent BABAR results on electroweak penguin and leptonic decays are reviewed. In particular, the measurements of $B \rightarrow K^{(*)} l^{+} l^{-}$and the preliminary results on $B \rightarrow X_{s} l^{+} l^{-}$are presented. Also summarized are the preliminary limits on $B^{+} \rightarrow l^{+} \nu(l=e, \mu)$ and $B^{+} \rightarrow K^{+} \nu \bar{\nu}$.
\end{abstract}

\section{Introduction}

All decays discussed in this review are sensitive to new physics ("NP"). The flavor changing neutral current decays, such as $b \rightarrow s l^{+} l^{-}$and $B^{+} \rightarrow K^{+} \nu \bar{\nu}$, do not occur at tree level and the $B^{+} \rightarrow l^{+} \nu(l=e, \mu)$ decays, which involve the $u \bar{b}$ annihilation diagram, are suppressed by the dependence on the CKM matrix element, $\left|V_{u b}\right|$, and the lepton mass, $m_{l}$. Hence, the branching fractions predicted by the Standard Model ("SM") for these decays are low and these are good channels to look for non-standard contributions with the potential for new particles to show up virtually.

\section{Electroweak Penguin Decays}

In the SM, the amplitudes which contribute to the $b \rightarrow s l^{+} l^{-}$and the $b \rightarrow s \nu \bar{\nu}$ decays at leading order are: the electromagnetic penguin (only for $b \rightarrow s l^{+} l^{-}$), the $\mathrm{Z}$ penguin and the $W^{+} W^{-}$box diagram. An important consequence of the loop structure of these decays is that their branching fractions and their kinematic variables, such as the transferred momentum squared of the virtual $\gamma / Z\left(q^{2}=M\left(l^{+} l^{-}\right)^{2}\right)$ and the forward-backward asymmetry of the lepton decay angle $\left(A_{F B}\right)$ in the $b \rightarrow s l^{+} l^{-}$decays, can be significantly affected by the presence of new particles or couplings predicted in non-standard scenarios. 


\section{1. $B \rightarrow K^{(*)} l^{+} l^{-}$}

The BABAR analysis ${ }^{1}$ is based on a data sample of $123 \cdot 10^{6} B \bar{B}$ pairs and studies eight final states: $B^{+} \rightarrow K^{+} l^{+} l^{-}, B^{0} \rightarrow K_{s}^{0} l^{+} l^{-}, B^{+} \rightarrow K^{*+} l^{+} l^{-}$ and $B^{0} \rightarrow K^{* 0} l^{+} l^{-}$, where $l$ is either an electron or a muon. $K^{(*)} e^{+} e^{-} \gamma$ events are also included in the $K^{(*)} e^{+} e^{-}$signal.

The exclusive reconstruction of the $\mathrm{B}$ mesons exploits the kinematic constraints on the beam-energy substituted mass $m_{E S}=\sqrt{E_{\text {beam }}^{* 2}-p_{B}^{* 2}}$ and $\Delta E=E_{B}^{*}-E_{\text {beam }}^{*}$, where $E_{\text {beam }}^{*}, p_{B}^{*}$ and $E_{B}^{*}$ are the beam energy, the momentum and the energy of the $\mathrm{B}$ candidate, respectively, in the center-of-mass ("CM") frame.

The $m_{E S}, \Delta E$ and $m_{K \pi}$ (only for the $B \rightarrow K^{*} l^{+} l^{-}$mode) distributions are simultaneously fitted to extract the signal (see Figure 1). Signals for
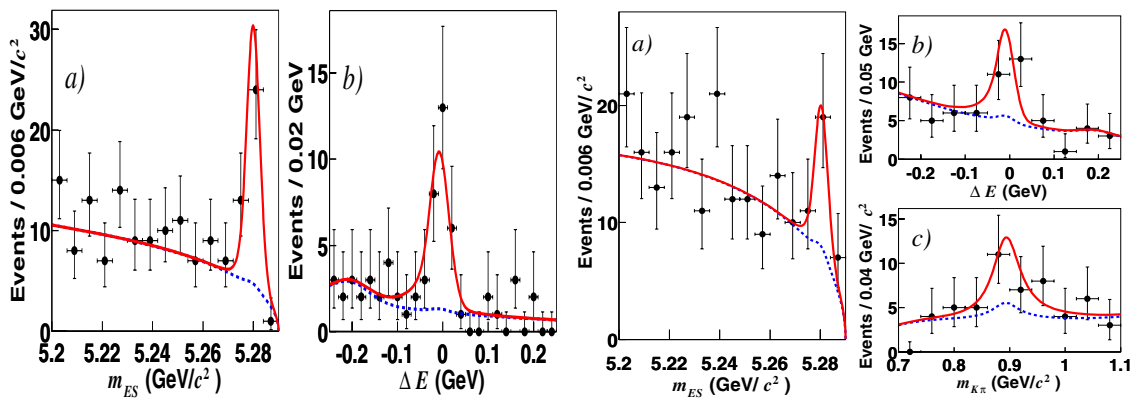

Figure 1. Distribution of $m_{E S}$ and $\Delta E$ in $B \rightarrow K l^{+} l^{-}$data (a) and $b$ ) on the left) and in $B \rightarrow K^{*} l^{+} l^{-}$data, where the $m_{K \pi}$ distribution is also shown $\left.(a), b\right)$ and $c$ ) on the right). The solid curves are the projections of the simultaneous fit. The dashed curves represent the background components under the signal peaks.

$B \rightarrow K l^{+} l^{-}$have been observed ( $\sim 8 \sigma$ of significance) and evidence for $B \rightarrow$ $K^{*} l^{+} l^{-}$has been obtained (3.3 $\sigma$ of significance). The measured branching fractions:

$$
\begin{aligned}
\mathcal{B}\left(B \rightarrow K l^{+} l^{-}\right) & =\left(0.65_{-0.13}^{+0.14} \pm 0.04\right) \cdot 10^{-6} \\
\mathcal{B}\left(B \rightarrow K^{*} l^{+} l^{-}\right) & =\left(0.88_{-0.29}^{+0.33} \pm 0.10\right) \cdot 10^{-6}
\end{aligned}
$$

are consistent with the range of predictions based on the SM.

\section{2. $B \rightarrow X_{s} l^{+} l^{-}$}

The $B \rightarrow X_{s} l^{+} l^{-}$branching fraction is computed in the SM with smaller theoretical uncertainties than $B \rightarrow K^{(*)} l^{+} l^{-}$. 
BABAR measured the $B \rightarrow X_{s} l^{+} l^{-}$branching fraction ${ }^{2}$ on a data sample of $89 \cdot 10^{6} B \bar{B}$ pairs by reconstructing the final state from pairs of electrons or muons and ten different hadronic states: $K^{+}, K^{+} \pi^{0}, K^{+} \pi^{-}$, $K^{+} \pi^{-} \pi^{0}, K^{+} \pi^{-} \pi^{+}, K_{s}^{0}, K_{s}^{0} \pi^{0}, K_{s}^{0} \pi^{+}, K_{s}^{0} \pi^{+} \pi^{0}$ and $K_{s}^{0} \pi^{+} \pi^{-}$.

A signal of $41 \pm 10$ (stat) \pm 2 (syst) events with a statistical significance of 4.6 was observed (see Figure 2). The branching fraction result:
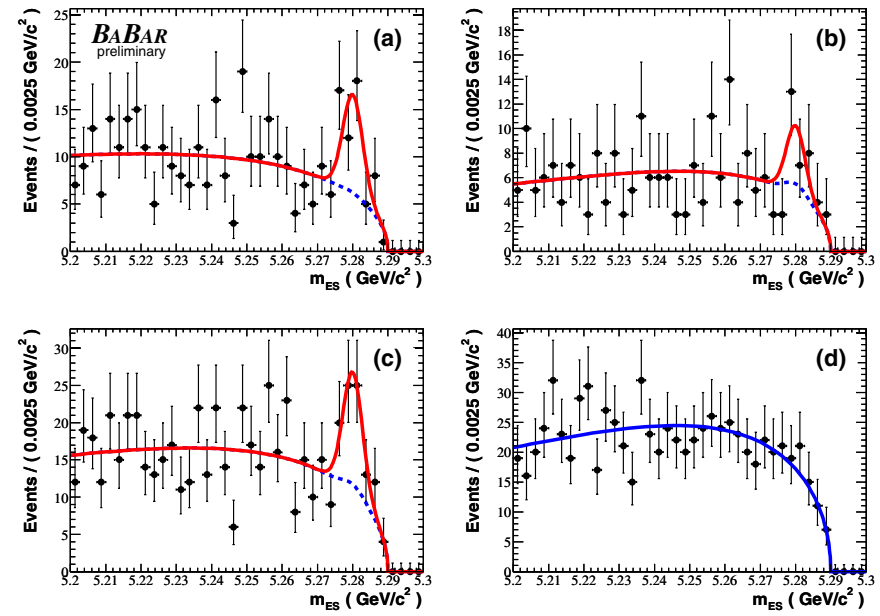

Figure 2. $m_{E S}$ distributions for (a) $B \rightarrow X_{s} e^{+} e^{-}$, (b) $B \rightarrow X_{s} \mu^{+} \mu^{-}$, (c) $B \rightarrow$ $X_{s} l^{+} l^{-}(l=e, \mu)$ and $(d) B \rightarrow X_{s} e^{+} \mu^{-}$candidates. The solid lines represent the result of the fits and the dashed lines the background components under the signal peaks.

$$
\mathcal{B}\left(B \rightarrow X_{s} l^{+} l^{-}\right)=\left(6.3 \pm 1.6_{-1.5}^{+1.8}\right) \times 10^{-6}
$$

agrees with the theoretical prediction by Ali et al. ${ }^{3}$ and the measurement performed by the Belle Collaboration ${ }^{4}$.

\section{3. $B^{+} \rightarrow K^{+} \nu \bar{\nu}$}

The $B^{+} \rightarrow K^{+} \nu \bar{\nu}$ decay measurement is experimentally challenging due to the presence of two unobserved neutrinos. To identify the signal, the other $\mathrm{B}$ in the event has to be fully reconstructed.

The BaBar analysis ${ }^{5}$, performed on a data sample of $87 \cdot 10^{6} B \bar{B}$ pairs, fully reconstructs the hadronic decay $B^{-} \rightarrow D^{0} X^{-}$of the other $\mathrm{B}$, where $X^{-}$is a hadronic sistem composed of up to three charged pions or kaons and 
up to two $\pi^{0}$ with a net charge of -1 . Candidates events are then required to possess only one kaon with $\mathrm{CM}$ momentum greater than $1.5 \mathrm{GeV} / \mathrm{c}$. Three candidates are found, which leads to an upper limit of $\mathcal{B}\left(B^{+} \rightarrow K^{+} \nu \bar{\nu}\right)<$ $10.5 \times 10^{-5}(90 \% \mathrm{CL})$. BaBar also set a preliminary limit of $9.4 \times 10^{-5}{ }^{5}$ by reconstructing the semileptonic decay of the other $\mathrm{B}$. The combined upper limit is:

$$
\mathcal{B}\left(B^{+} \rightarrow K^{+} \nu \bar{\nu}\right)<7.0 \times 10^{-5}(90 \% C L)
$$

which is still an order of magnitude higher than the SM prediction $\mathcal{B}\left(B^{+} \rightarrow K^{+} \nu \bar{\nu}\right)=\left(3.8_{-0.6}^{+1.2}\right) \cdot 10^{-6}$.

\section{Leptonic Decays}

The study of the purely leptonic $B^{+} \rightarrow l^{+} \nu$ decays can provide sensitivity to some SM parameters and also act as a probe of NP.

In the SM, the branching fraction can be cleanly computed:

$$
\mathcal{B}\left(B^{+} \rightarrow l^{+} \nu\right)=\frac{G_{F}^{2} m_{B} m_{l}^{2}}{8 \pi}\left(1-\frac{m_{l}^{2}}{m_{B}^{2}}\right) f_{B}^{2}\left|V_{u b}\right|^{2} \tau_{B}
$$

where $G_{F}$ is the Fermi coupling constant, $m_{l}$ and $m_{B}$ are the charged lepton and $\mathrm{B}$ meson masses, $\tau_{B}$ is the $B^{+}$lifetime and $f_{B}$ the $\mathrm{B}$ decay constant. Using data from Ref. 7 the SM expectations are $\mathcal{B}\left(B^{+} \rightarrow \tau^{+} \nu\right) \sim 7.5 \times 10^{-5}$ and $\mathcal{B}\left(B^{+} \rightarrow \mu^{+} \nu\right) \sim 4 \times 10^{-7}$.

A measurement could provide either the first direct determination of $f_{B}$, given the CKM matrix element $\left|V_{u b}\right|$, or evidence for NP since a non SM amplitude could enhance the predicted branching fraction.

\section{1. $B^{+} \rightarrow \tau^{+} \nu$}

BABAR has searched the $B^{+} \rightarrow \tau^{+} \nu$ decay on a data sample of $89 \cdot 10^{6}$ $B \bar{B}$ pairs. Due to the presence of at least two neutrinos in the final state, the semileptonic and the hadronic decays of the other $B$ have been reconstructed, as in the $B^{+} \rightarrow K^{+} \nu \bar{\nu}$ analysis.

After reconstructing the other $\mathrm{B}$, the signal signature is given by one or up to tree charged tracks, depending on the $\tau$ decay mode, and low neutral energy left in the detector $\left(E_{l e f t}\right)$. In the analysis using the semileptonic $\operatorname{tag}^{8}$, only the $\tau^{+} \rightarrow e^{+} \nu_{e} \bar{\nu}_{\tau}$ and $\tau^{+} \rightarrow \mu^{+} \nu_{\mu} \bar{\nu}_{\tau}$ decays are reconstructed and a fit to $E_{\text {left }}$ is performed. In the analysis using the hadronic tag ${ }^{9}$, the hadronic $\tau$ decays into $\pi^{+} \bar{\nu}_{\tau}, \pi^{+} \pi^{0} \bar{\nu}_{\tau}$ and $\pi^{+} \pi^{-} \pi^{+} \bar{\nu}_{\tau}$ are also included 
and the number of events with the $E_{\text {left }}<100 \mathrm{MeV}$ is counted. The two samples are then combined to obtain:

$$
\mathcal{B}\left(B^{+} \rightarrow \tau^{+} \nu\right)<4.1 \times 10^{-4}(90 \% C L)
$$

which improves the upper limit given by L3 ${ }^{10}$.

\section{2. $B^{+} \rightarrow \mu^{+} \nu$}

The $B^{+} \rightarrow \mu^{+} \nu$ decay has been searched by BaBar on a data sample of $88 \cdot 10^{6} B \bar{B}$ pairs. ${ }^{11}$ After identifying a muon, all remaining particles are associated with the decay of the other B. Once the other B is reconstructed, the muon momentum is calculated in the rest frame of the signal $\mathrm{B}$. The signal muon momentum distribution is expected to peak at $2.64 \mathrm{GeV} / \mathrm{c}$. No significant signal excess has been observed and the obtained upper limit:

$$
\mathcal{B}\left(B^{+} \rightarrow \mu^{+} \nu\right)<6.6 \times 10^{-6}(90 \% C L)
$$

is the most stringent upper limit set so far for the $B^{+} \rightarrow \mu^{+} \nu$ branching fraction.

\section{Conclusion}

No deviations from the SM have been found in electroweak and leptonic B decays, yet, but limits on several exclusive modes have been improved and the observation of $B \rightarrow K^{*} l^{+} l^{-}$and $B \rightarrow X_{s} l^{+} l^{-}$opens the way to a new rich area of search for new physics.

\section{References}

1. B. Aubert et al., BABAR Collaboration, arXiv:hep-ex/0308042;

2. B. Aubert et al., BABAR Collaboration, arXiv:hep-ex/0308016;

3. A. Ali, E. Lunghi, C. Greub and G. Hiller, Phys. Rev. D66, 034002 (2002);

4. J. Kaneko et al., Belle Collaboration, Phys. Rev. Lett. 90, 021801 (2003);

5. B. Aubert et al., BABAR Collaboration, arXiv:hep-ex/0304020;

6. B. Aubert et al., BABAR Collaboration, arXiv:hep-ex/0207069;

7. Particle Data Group, Phys. Rev. D66, 010001 (2002);

8. B. Aubert et al., BABAR Collaboration, arXiv:hep-ex/0303034;

9. B. Aubert et al., BABAR Collaboration, arXiv:hep-ex/0304030;

10. M.Acciarri et al., L3 Collaboration, Phys. Lett. B396, 327-337 (1997);

11. B. Aubert et al., BABAR Collaboration, arXiv:hep-ex/0401002; 